\title{
Effects of the Allelochemicals Dihydrodiconiferyl Alcohol and Lariciresinol on Metabolism of Lactuca sativa
}

\author{
Petronia Carillo ${ }^{1, *}$, Concettina Cozzolino ${ }^{1}$, Brigida D’ Abrosca ${ }^{1}$, Francesco Nacca ${ }^{1}$, \\ Marina DellaGreca ${ }^{2}$, Antonio Fiorentino ${ }^{1}$ and Amodio Fuggi ${ }^{1}$ \\ ${ }^{1}$ Dipartimento di Scienze della Vita - Seconda Università di Napoli - via Vivaldi 43, 81100 Caserta, Italy \\ ${ }^{2}$ Dipartimento di Chimica Organica e Biochimica, Università Federico II, via Cynthia 4, 80126 Napoli, Italy
}

\begin{abstract}
Dihydridiconiferyl alcohol and lariciresinol, known as phytotoxic lignans, acting on the germination of Lactuca sativa have been tested for lettuce seedling development. It has been evidenced that sucrose grown plants respond to such lignans differently from those grown without sucrose. Dihydridiconiferyl alcohol, which is more active than lariciresinol, reduced chlorophyll and carotenoid synthesis in sucrose grown plants. It also caused a greater decrease of photosystem efficiency, lowering of Yield and non-photochemical quenching in treated plants in respect to those in controls. Both lignans affected carbon and nitrogen metabolism, reducing, in particular, glucose-6-phosphate dehydrogenase (G6PDH) and glutamate synthase (GOGAT) activities in sucrose grown plants. The decrease of G6PDH (-80\%) and GOGAT $(-65 \%)$ activities was correlated to an increase of soluble sugars (up to $+100 \%)$ and glutamine $(+70 \%)$, respectively. This is the first time an allelochemical affecting GOGAT activity has been reported.
\end{abstract}

Keywords: Lactuca sativa, Lignans, Dihydridiconiferyl alcohol, Lariciresinol, NPQ, G6PDH, GOGAT, Glutamine, Carbohydrates.

\section{INTRODUCTION}

Allelopathy is the chemical interaction between plants. Plant species synthesize chemicals that are spread into their surroundings and can affect other plants. Generally, such metabolites, identified as allelochemicals, diffuse from producing plants to recipient plants. This means that the amount of toxin around the donor plant will be higher than that around the recipient plant, and suggests that the donor plant must have evolved resistance to the toxin [1].

In recent years, allelochemicals have been well investigated as a source of new molecules for the chemical weed control [2]. Herbicides have played an important role in agricultural practices and their use has allowed high yields of crop, reducing both the efforts of the laborer and the cost of production. Nevertheless, the massive use of synthetic herbicides has caused weeds to evolve resistance to many of them, so stimulating the search for new, synthetic herbicides with different target sites [3]. Rotational or smoother crops such as rye, wheat, clack mustard are usually used in weed management and many compounds isolated from these plants are reported as potential allelochemicals. Research in this field has led to the identification and development of cinmethylin from cineol, a natural terpene [4]. Allyl isothiocyanate, fatty acids, isoflanonoids, phenols and phenolic acids are examples of allelopathic compounds that are reported to play a role in weed management [5]. Thus,

*Address correspondence to this author at the Dipartimento di Scienze della Vita - Seconda Università di Napoli - via Vivaldi 43, 81100 Caserta, Italy; Tel: +39 0823274562; Fax: +390823274571;

E-mail: petronia.carillo@unina2.it in the search for new friendly molecular skeletons, allelochemicals represent an inexhaustible source of natural compounds to be utilized as alternatives to synthetic ones.

Several classes of allelochemicals, such as terpenoids, phenols and alkaloids, have been isolated in recent years. Duke et al. (2002) reviewed strategies for the discovery of natural compounds such as templates of herbicides and Macias et al. (2000) proposed a collection of Standard Target Species (STS) of commercial crops as models for weed control [6,7].

In the search for allelochemicals, such as herbicides or templates for new herbicides classes from plants, we have studied plants of the Mediterranean area. Many potential allelochemicals have been isolated and characterized. We isolated some bioactive cyanogenic glycosides from Sambucus nigra [8] and phytotoxic low molecular weight phenols from Cestrum parqui [9]. Interesting results have been obtained in the phytochemical study of Brassica fruticulosa, a plant belonging to the large family of Brassicaceae and widely distributed in the Mediterranean area. The plant infusions resulted active when assayed on Lactuca sativa L. (lettuce) and the study of active extracts led to the isolation of five lignans, five neolignans, two sesquilignans and a dilignan [10]. Lignans are widely distributed in plants and derived from the phenylpropanoid pathway. These compounds exhibit interesting antimicrobic, antiviral, herbicidal, or antifeedant activities that are thought to participate in plant defence mechanisms against biotic stresses [11, 12]. The cancer protective effects of dietary lignans have been also demonstrated [13] but not many data are available regarding 
the phytotoxic activity of this class of compounds or their ability to interact with other plant organisms.

Among the compounds isolated from Brassica fruticulosa, dihydrodiconiferyl alchohol (1) and lariciresinol (2) (Fig. 1), were indeed very interesting both for their high concentrations in the producing plant and for their inhibiting effect on the germination of lettuce [10]. Their bioactivity and high concentration allowed us to plan experiments on the effect of such compounds on Lactuca sativa L seedling metabolism.

\section{MATERIAL AND METHODS}

\section{Plant Material and Growth Conditions}

Lettuce seeds (Lactuca sativa) were surface-sterilized by immersion in $1.25 \%$ sodium hypochlorite for 1 minute, immersed in sterile water for $1 \mathrm{~h}$, and subsequently in sterile 2 $\mathrm{mM} \mathrm{CaSO}_{4}$ for $1 \mathrm{~h}$, followed by three washes in sterile distilled water. Seeds were germinated on filter paper moistened with deionised water in darkness at $21{ }^{\circ} \mathrm{C}$. After 4 days, individual lettuce seedlings were immersed in $1.25 \%$ sodium hypochlorite for a few seconds, washed again in sterile distilled water and then transferred to thirty $100-\mathrm{mL}$ sterilized pots (15 plants per pot) containing $4.4 \mathrm{~g} / 1$ Murashige and Skoog basal medium (MS) (Sigma) solidified with $10 \mathrm{~g} / \mathrm{l}$ agar (Sigma). Eighteen pots contained sucrose to final concentration of $88 \mathrm{mM}$. The control plants in the other twelve pots were grown without supplemented sucrose.

\section{Experimental Design for Lignan Treatments}

Plants were kept under controlled conditions (16 h photoperiod, $300 \mu \mathrm{mol} \mathrm{m}{ }^{-2} \mathrm{~s}^{-1} \mathrm{PAR}$, thermoperiod $25 / 20{ }^{\circ} \mathrm{C}$ day/night, $65 \%$ relative humidity).

Preliminary experiments were carried out at four different dihydrodiconiferyl alchohol (1) and lariciresinol (2) concentrations $\left(10^{-4}, 10^{-6}, 10^{-8}\right.$ and $\left.0 \mathrm{M}\right)$ with and without sucrose. Since lariciresinol was less active than dehydriconiferyl alcohol, in the final experimental design, on day four of MS medium culture, plants were divided into 10 groups (three pots for each group), adding dehydroconiferil alcohol dissolved in DMSO up to a concentration of $10^{-4}$, $10^{-6}$ and $10^{-8} \mathrm{M}$ and lariciresinol dissolved in DMSO, up to concentrations of $10^{-4}$ and $10^{-6} \mathrm{M}$. Treatments with dehydroconiferil alcohol were performed with or without sucrose. Controls, with and without sucrose, were treated only with DMSO.

After 14 days of MS medium culture, $4 \mathrm{~h}$ after the beginning of the light period, the lengths of the plants were meas-

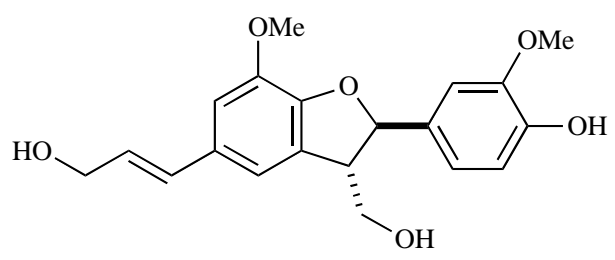

ured, fluorescence measurements were performed and then plants from each pot were harvested, ground in liquid nitrogen and either used immediately for assays or stored at -80 ${ }^{\circ} \mathrm{C}$ for further analysis. Aliquots of these materials were used to determine chlorophylls, carotenoids, soluble and total carbohydrates and enzyme activities.

Before harvesting fluorescence measurements were performed on growing plant leaves.

\section{Plant Analysis}

\section{Fluorescence and Chlorophyll Content}

Maximum quantum efficiency of Photosystem II (Yield) and non-photochemical dissipation of absorbed light energy (NPQ) were measured with a portable pulse-modulated fluorometer (PAM-2000 Walz, Effeltrich, Germany) in accordance with Deltoro et al. (1999) [14]. Photosynthetic pigments were extracted in Dimethyl-formamide and measured in accordance with Wellburn (1994) [15].

\section{Protein Determinations}

Protein concentration was quantified by using the Bio-Rad protein essay based on the Bradford method [16] with bovine serum albumin as standard. Soluble and total protein contents were quantified by the Bradford method with bovine serum albumin as standard as described in Augusti et al. (1999) [17].

\section{Solute Analysis}

Amino acids were extracted and assayed as described by Carillo et al. (2005) [18], mixing aliquots of $50 \mathrm{mg}$ fresh wt with $1 \mathrm{ml}$ of EtOH: $\mathrm{H}_{2} \mathrm{O}(40: 60 \mathrm{v} / \mathrm{v})$. The primary amino acids were determined by HPLC after precolumn derivatization of extract with OPA reagent. Proline was extracted and estimated as described by Carillo et al. (2008) [19]. Sugars and starch were extracted and determined in accordance with Pietrini et al. (1999) [20].

\section{Enzyme Extraction and Assays}

Enzymes were extracted and assayed as described in the following references: Glutamine synthetase (GS) [21], Aspartate amino transferase (AspAT), Glutamate synthase (GOGAT), Glutamate dehydrogenase (GDH) and Phosphoenolpyruvate carboxylase (PEPcase) [22], Glucose-6phosphate dehydrogenase (G6PDH) [23].

\section{RESULTS}

Biotests were carried out on lettuce plants grown on MS complete medium with sucrose, supplemented with the pure<smiles>COc1cc(C[C@H]2CO[C@H](c3ccc(O)c(OC)c3)[C@H]2CO)ccc1O</smiles>

Fig. (1). Chemical structures of dihydrodiconiferyl alcohol (1) and lariciresinol (2). 
lignans dissolved in DMSO. Compound 1 was tested at the $10^{-4}, 10^{-6}$ and $10^{-8} \mathrm{M}$ concentrations, while compound 2 at $10^{-4}$ and $10^{-6} \mathrm{M}$. Only compound 1 was tested also in MS medium without sucrose.

Chlorophylls, total carotenoids, Yield, NPQ, carbohydrates, proteins, free amino acids and activity of enzymes of carbon and nitrogen metabolism were evaluated in the leaves of ten-day-treated plants and compared with the controls supplemented only with DMSO.

Fig. (2A) shows that only in complete Murashige and Skoog medium supplemented with sucrose did the lignan treatment reduce the leaf pigment content to about $61 \%$ and $83 \%$ of the controls, respectively in presence of compounds 1 and 2 , even at $10^{-6} \mathrm{M}$. Chlorophyll a and carotenoids were less affected by lignans treatment than chlorophyll b (Figs. 2 B-D). Only for lignan 1 related to the pigment content we found an effect not dose-dependent. We could not explain this result. Such treatment increased the level of chlorophylls and carotenoids up to $185 \%$ in comparison with its control, when seedlings were grown in MS without sucrose (Figs. 2 A-D).

As the decrease of pigment concentration was greater for compound $\mathbf{1}$ than for compound $\mathbf{2}$, with the former active also at $10^{-8} \mathrm{M}$, compound $\mathbf{1}$ was used for chlorophyll fluorescence analysis to evaluate the photosystem efficiency.

In Fig. (3), already at $10^{-8} \mathrm{M}$, compound $\mathbf{1}$ adversely affected Yield and non-photochemical quenching of absorbed light energy (NPQ) in the leaves of lettuce plants. In plants grown on sucrose MS medium, the Yield, related to the maximum quantum efficiency of photosystem II, decreased by $17 \%$ with respect that in the control; in the same leaves

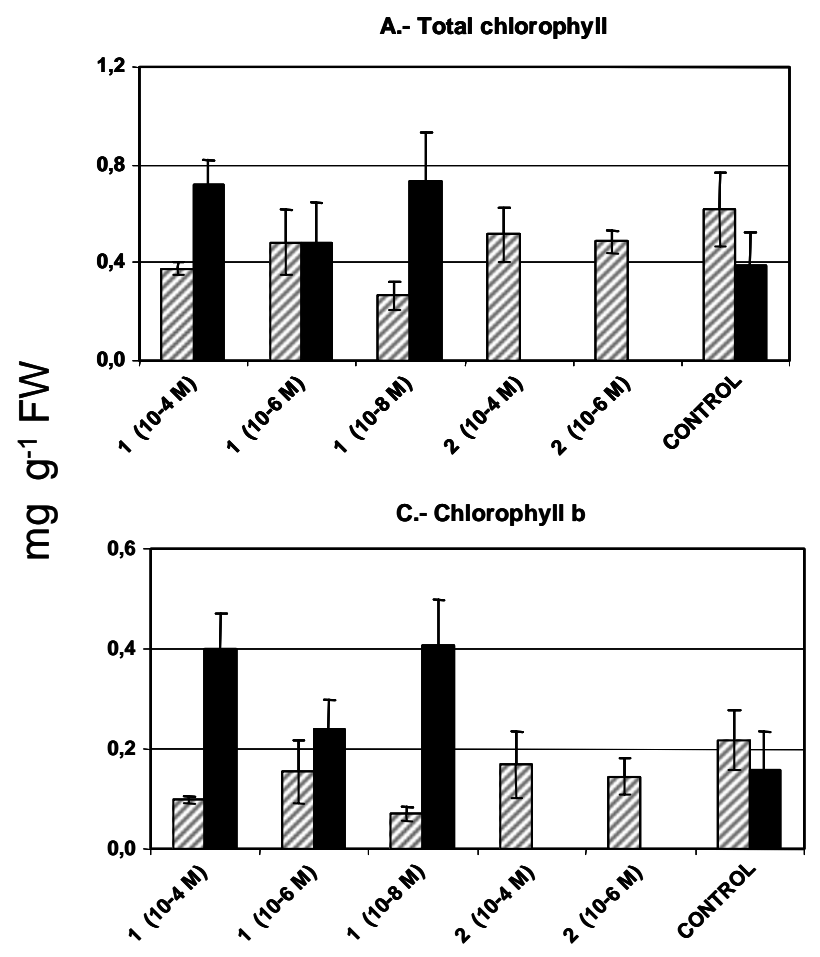

NPQ, the index of the capacity for photoprotective processes [24], declined by $66 \%$ in comparison with that in the control. On the contrary, neither Yield nor NPQ evidenced very significant change in the leaves of recipient plants grown without sucrose.

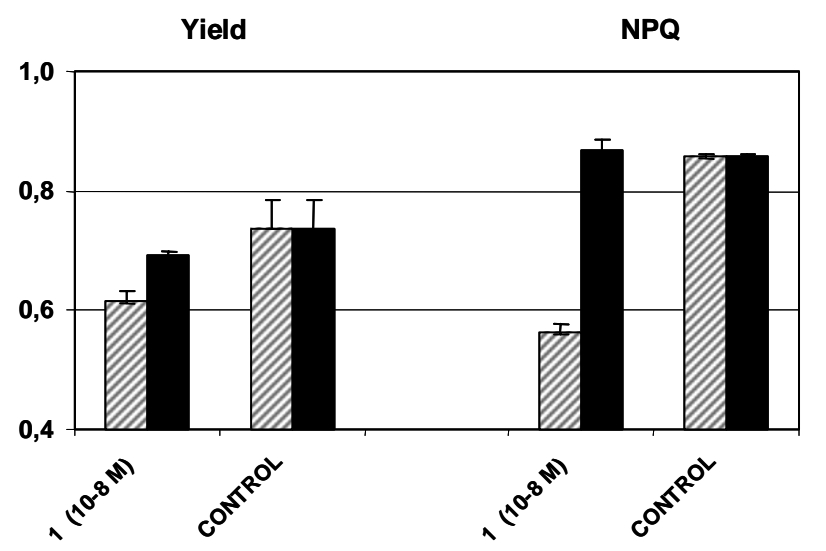

Fig. (3). Effect of dihydrodiconiferyl alcohol (1) dissolved in DMSO on Yield and NPQ in leaves of lettuce after 14 days of MS medium culture, supplemented with ( $\square$ ) or without sucrose ( $\square$ ). Lignans were added from the day 4 of MS culture. Controls, with and without sucrose, were treated only with DMSO. The values are mean $\pm \operatorname{SD}(n=6)$.

Damage or inhibition of glycolytic and/or oxidative penthose phosphate pathway (OPPP) enzymes have often been related to the inhibition of seed germination by allelochemicals [25]. Thus Fig. (4A) shows the activity of glucose-6-phosphate dehydrogenase (G6PDH) highly decreased

\section{B.- Chlorophyll a}

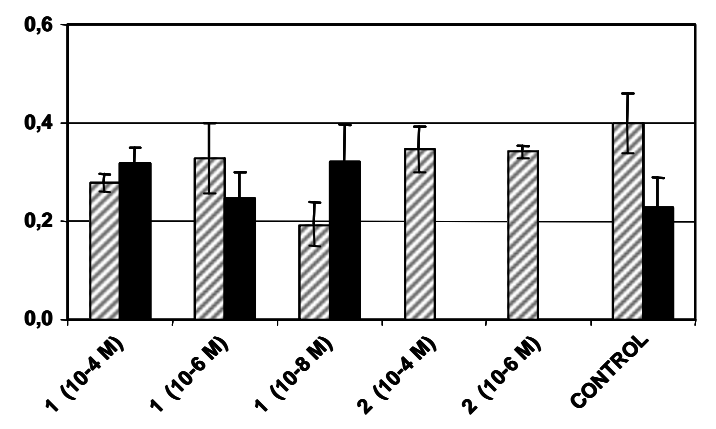

D.- Total carotenoids

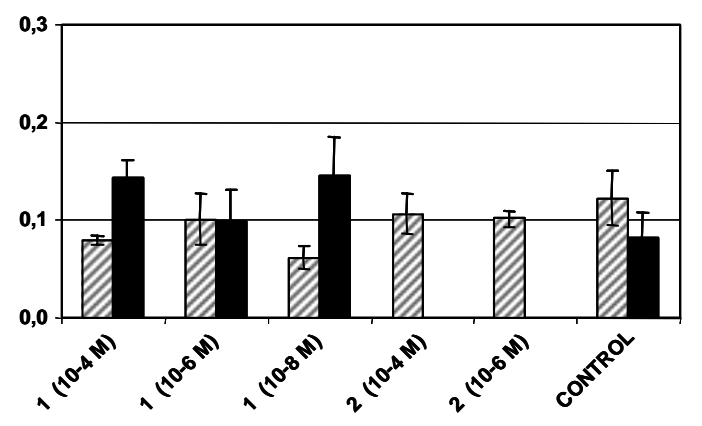

Fig. (2). Effect of dihydrodiconiferyl alcohol (1) and lariciresinol (2) dissolved in DMSO on pigments content in leaves of lettuce after 14 days of MS medium culture, supplemented with ( or without sucrose ( $)$. Lignans were added from the day 4 of MS culture. Controls, with and without sucrose, were treated only with DMSO. The values are mean $\pm \operatorname{SD}(n=4)$. 


\section{A- G6PDH}

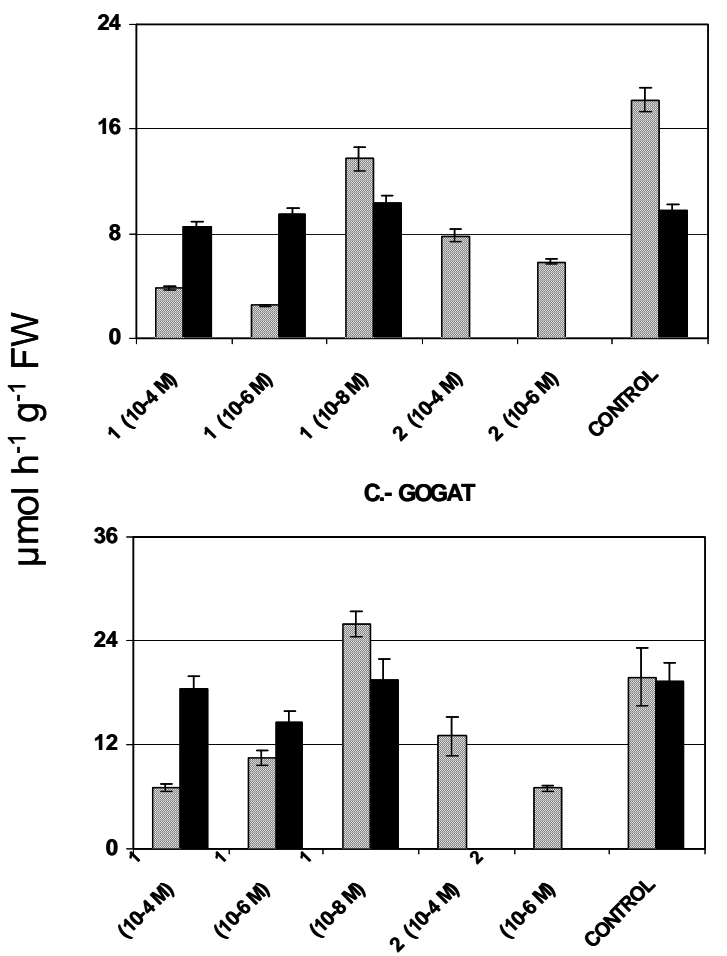

B.- GS

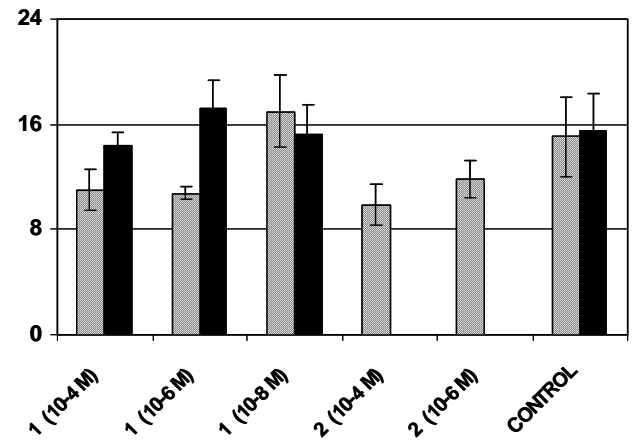

D.- GDH

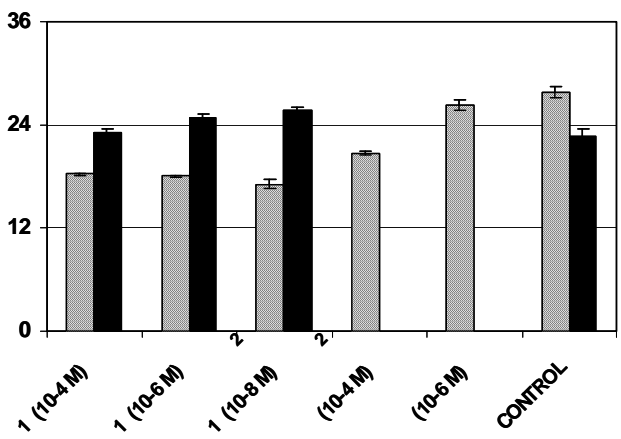

Fig. (4). Effect of dihydrodiconiferyl alcohol (1) and lariciresinol (2) dissolved in DMSO on the activities of carbon and nitrogen metabolism enzymes in lettuce leaves after 14 days of MS medium culture, supplemented with ( $)$ or without sucrose ( $\square$ ). Lignans were added from the day 4 of MS culture. Controls, with and without sucrose, were treated only with DMSO. The values are mean \pm SD $(n=4)$.

under lignan treatment, but only in the leaves of sucrose grown plants. Compounds 1 and 2 , at $10^{-6} \mathrm{M}$, reduced leaf average G6PDH activity respectively to about $20 \%$ and $40 \%$ of the controls. Instead, in plants grown without sucrose the G6PDH activity was not significantly inhibited (13\%) even at the highest compound $\mathbf{1}$ concentration tested with respect to the control (54\% of control with sucrose). The enzymes of nitrogen metabolism were also inhibited by lignan treatment in sucrose grown plants even at $1 \mu \mathrm{M}$ : GS (Fig. 4B) and GOGAT (Fig. 4C) (the essential enzymes for fixing ammonia into amino acids) were decreased on average by $30 \%$ and $65 \%$ in the controls, respectively, as with GDH, which decreased by $35 \%$, too. While lignan 1 was more active than lignan 2 in inhibiting G6PDH and GDH activities, the effect of the two lignans on GS and GOGAT was comparable. No effect was evidenced in plants grown without sucrose.

Other tested enzymes, PEP carboxylase and aspartate amino transferase were not significantly affected by lignan in any of the treatments (not shown).

Figs. (5A-B) show total and soluble proteins which also decreased in leaves of plants grown with sucrose after lignan treatment. At $10^{-6} \mathrm{M}$, the average total and soluble proteins were $95 \%$ and $75 \%$ of those for controls, respectively. In cultures without sucrose, total proteins were on average about $60 \%$ of those of the controls with sucrose, but did not change significantly; whilst soluble proteins $(86 \%$ of controls with sucrose) decreased by up to $33 \%$ in respect to their control.

Free amino acids content (Fig. 5C) increased (about $40 \%$ ) in leaves of sucrose grown plants. Among the free amino acids, glutamine, the most abundant amino acid in lettuce ( $54 \%$ of the total), increased by $70 \%$ in comparison with that in control, reaching $65 \%$ of the total. In cultures without sucrose, free amino acids did not change, but the average glutamine content (11\% of the control with sucrose) decreased to $8 \%$ of total free amino acids, irrespective of lignan treatment.

Carbohydrates (Fig. 6A) also increased in leaves of lignan treated sucrose grown plants. At $10^{-6} \mathrm{M}$ of compounds $\mathbf{1}$ and 2, their total content was $70 \%$ greater than that of the controls. In plants grown without sucrose, carbohydrates content was $20 \%$ that of the control with sucrose, but it did not change upon lignan treatment.

Starch controls (Fig. 6B) in leaves of plant grown with and without sucrose were $10 \%$ and $77 \%$ of total carbohydrates, respectively. Only in sucrose grown plants did lignan increase starch content up to $40 \%$ with respect to the control.

Sucrose (Fig. 6C) and reducing sugars (Fig. 6D), which were present in high concentrations only in plants supplemented with sucrose, increased by $60 \%$ and $100 \%$, respectively, under treatment with compounds $\mathbf{1}$ and $\mathbf{2}$.

\section{DISCUSSION}

Bioactive natural products from Mediterranean plant and their potential use as natural herbicide models have been investigated in Brassica fruticulosa Cyr., a plant widely distributed in the Mediterranean area. A previous report showed that the aqueous extracts of this plant resulted phytotoxic on the germination of Lactuca sativa L. (lettuce), and the study 

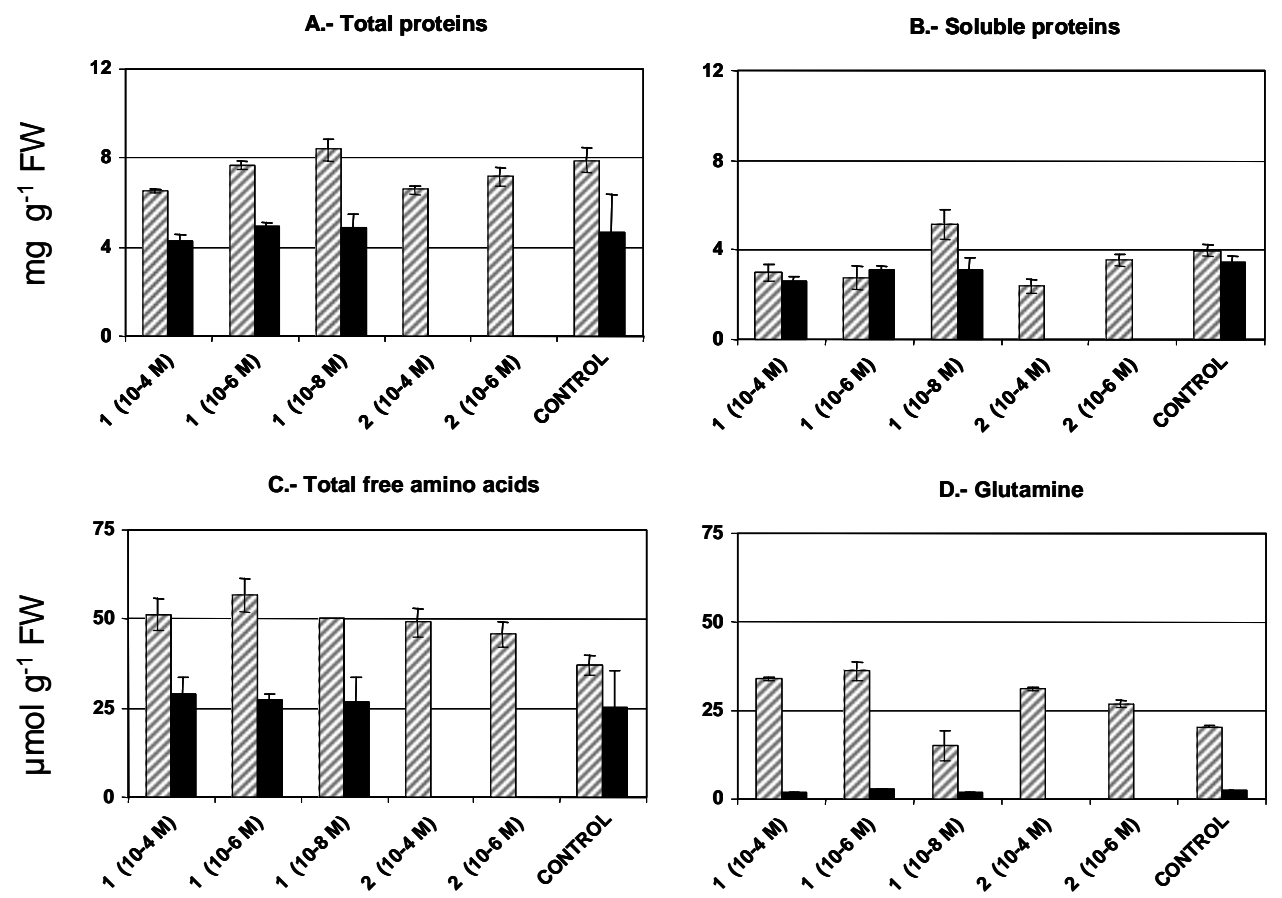

Fig. (5). Effect of dihydrodiconiferyl alcohol (1) and lariciresinol (2) dissolved in DMSO on protein and amino acid content in lettuce leaves after 14 days of MS medium culture, supplemented with $(\mathbb{Z})$ or without sucrose $(\square)$.ignans were added from the day 4 of MS culture. Controls, with and without sucrose, were treated only with DMSO. The values are mean \pm SD $(n=4)$.

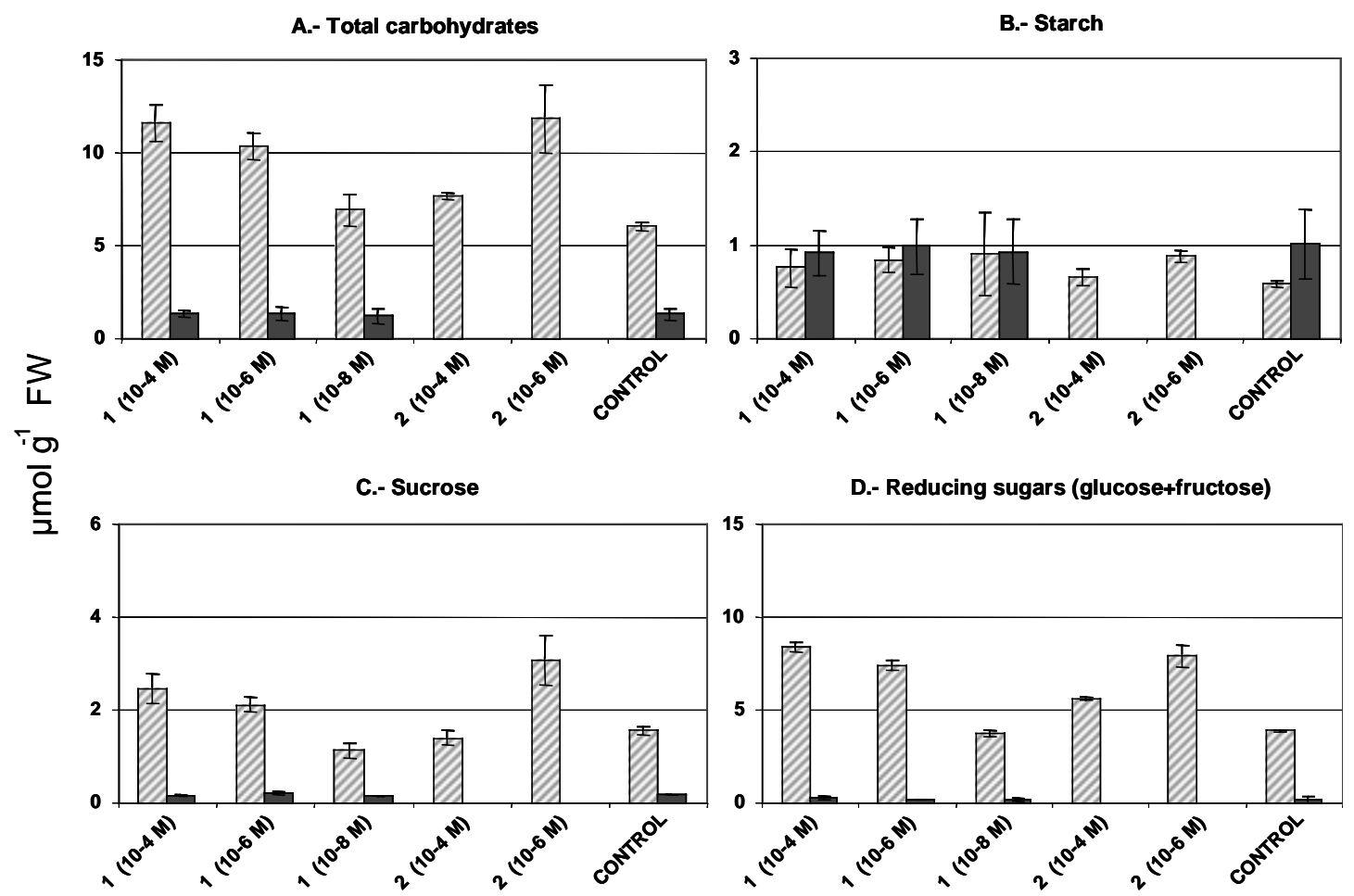

Fig. (6). Effect of dihydrodiconiferyl alcohol (1) and lariciresinol (2) dissolved in DMSO on carbohydrates content in lettuce leaves after 14 days of MS medium culture, supplemented with $(\llbracket$ )or without sucrose $(\square)$. Starch is expressed as glucose equivalents. Lignans were added from the day 4 of MS culture. Controls, with and without sucrose, were treated only with DMSO. The values are mean \pm SD ( $n=4$ ).

of active extracts led to the isolation of lignans, neolignans, sesquilignans and dilignan [10]. Among the bioactive lignans isolated, dihydrodiconiferyl alcohol (1) and lariciresinol (2) were indeed interesting both for their high concentrations in the producing plant and for their inhibiting effect on the germination of lettuce. 
Thus, we chose to examine the effect of these natural herbicides on several physiological and biochemical parameters of lettuce seedlings for identifying possible target sites of their action.

It was evident that plants grown in sucrose MS medium responded very differently to the lignan treatment compared to those grown without sucrose.

It is known that sugars repress the transcription of many genes involved in photosynthesis, and in particular pigment synthesis [26]. Fig. (2) shows that this is not the case of the lettuce in which chlorophylls and carotenoids, in plants grown on sucrose supplemented medium, were about $60 \%$ greater than those present in plants grown without sucrose. Treatment with compound $\mathbf{1}$, however, caused a decrease of pigment content in sucrose grown plants, but an increase in those grown autotrophycally. It might be suggested that the lignan can mediate the sucrose in repressing genes involved in the pigment synthesis in lettuce.

In nonvascular and vascular plants, chlorophyll b is one of the light harvesting pigments that function to bring photons to the reaction centers. Chl $\mathrm{b}$ binds and stabilizes many of the light-harvesting complex (LHC) proteins but is not associated with the reaction center or the core of photosystems PSI and PSII [27]. The $\mathrm{Chl} \mathrm{a} / \mathrm{b}$ ratio is positively correlated with the ratio of PSII cores to light harvesting chlorophyll-protein complex (LHCII), and an increase of this ratio, due to a decrease of chlorophyll b compared to chlorophyll a (Figs. 2B-D), could indicate that the light-harvesting antenna size is smaller. LHCII is also involved in scavenging reactive oxygen species, for this reason a smaller size entails a lower efficiency in contrasting photooxidative stress. In fact, the decrease of NPQ and Yield in neolignan $\mathbf{1}$ treated sucrose grown plants (Fig. 3) suggested that photoprotective processes were less efficient in quenching excess light energy, so causing damage to the photosynthetic apparatus [28].

This is, of course, reliant on the belief that the action of neolignan 1 was not due to a direct effect on electronic photosynthetic transport (as the one exercised by DCMU), to which, in this case, plants would be less sensitive, but to an action exercised by photosensitiving plants, which probably, mostly in the chloroplast, increase the production of hydrogen peroxide, singlet oxygen or other toxic substances, usually detoxified by some components of photosynthetic apparatus such as ascorbate peroxidase or carotenoids.

Both lignans reduced the activities of the carbon and nitrogen metabolism enzymes in plants grown on sucrose MS medium. Compounds $\mathbf{1}$ and $\mathbf{2}$, in fact, strongly reduced G6PDH activity (Fig. 4A), and this was positively correlated to an increase of soluble sugars (sucrose, glucose and fructose) (Figs. 6C-D). No evidence had been reported previously that this enzyme was inhibited in seedlings. This suggested that also in seedlings lignans could severely affect OPPP enzymes, and not only during seed germination, as generally reported for allelophatic chemicals [29].

Lignans strongly reduced the activity of GOGAT, much more than that of GS, but only in sucrose grown plants. The data were supported by the fact that glutamine, which represented $54 \%$ of the free amino acids in lettuce, further increased $(+70 \%$ that the control) (Fig. 5D). It might be suggested that the main effect of lignans was on GOGAT enzyme. The inhibition of GS could be related to the increase of glutamine, the allosteric effector of GS. The GDH activity was also inhibited in such plants: the average deaminating activity was strongly reduced (- $47 \%$ of control), whilst the average aminating activity decreased to a lesser extent (-17\% of control) (not shown) (Fig. 4D).

Lettuce is a C3 plant, in which photorespiration occurs, the inhibition of GS and GOGAT activities, enzymes also involved in the reassimilation of ammonia released during photorespiration, could lead to an accumulation of ammonia into chloroplast and a subsequent leaf damage.

\section{CONCLUDING REMARKS}

Our data suggested that, after treatment with these lignans, lettuce plants having a lower activity of oxygen-free radical scavenging, because of a smaller size of LHCII, were less tolerant to environmental stresses, showing damages that in other plants would have appeared only in presence of high intensity light. In addition, we have seen that lignans might be strong repressors of GOGAT activity: before this case, no herbicidal GOGAT inhibitors had been found $[29,30]$.

$\begin{array}{ll}\text { ABBREVIATIONS } \\ \text { GS } & =\text { Glutamine synthetase } \\ \text { GOGAT } & \text { Glutamate synthase } \\ \text { GDH } & =\text { Glutamate dehydrogenase } \\ \text { G6PDH } & =\text { Glucose-6-phosphate dehydrogenase } \\ \text { MS } & =\text { Murashige and Skoog basal medium } \\ \text { Yield } & =\text { Maximum quantum efficiency of Photosys- } \\ \text { NPQ } & =\text { Non-photochemical dissipation of absorbed } \\ \text { OPPP } & =\text { light energy }\end{array}$

\section{REFERENCES}

[1] Fitter, A. M. Ecology, making allelophathy respectable. Science, 2003, 301, 1337-1338.

[2] Vyvyan, J. A. Allelochemicals as leads for new herbicides and agrochemicals. Tetrahedron, 2002, 58, 1631-1646.

[3] Magnus, W. W. Structure-based design of agrochemicals. Nat. Prod. Rep., 2002, 19, 278-291.

[4] Jones, R. G. Cinmethylin- A new hernbicide developed for use in rice. In: Grayson, B. T., Green, M B., Copping L. G., Eds.; In: Pest Management in Rice. Elsevier: Amsterdam, 1990, p. 349.

[5] Bhowmik, P. C. Inderjit, Challenges and opportunities in implementing allelopathy for natural weed management. Crop Prot., 2003, 22, 661-671.

[6] Duke, S. O.; Dayan, F. E.; Rimando, A. M.; Schrader, K. K.; Aliotta, G.; Oliva, A.; Romagni, J. G. Chemicals from nature for weed management. Weed Sci., 2002, 50, 138-151.

[7] Macias, F. A., Castellano, D., Molinillo, J. M. G. Search for a standard phytotoxic bioassay for allelochemicals. Selection of standard target species. J. Agric. Food Chem., 2000, 48, 2512-2521.

[8] D'Abrosca, B.; DellaGreca, M.; Fiorentino, A.; Monaco, P.; Previtera, L.; Simonet, A. M.; Zarrelli, A. Potential allelochemicals from Sambucus nigra. Phytochemistry, 2001, 58, 1073-1081.

[9] D'Abrosca, B.; Della Greca, M.; Fiorentino, A.; Monaco, P.; Zarrelli. A. Low molecular weight phenols from bioactive aqueous fraction of Cestrum parqui. J. Agric. Food Chem., 2004, 52, 41014108 . 
[10] Cutillo, F.; D’Abrosca, B.; DellaGreca, M.; Fiorentino, A.; Zarrelli, A. Lignans and neolignans from Brassica fruticolosa: effects on seed germination and plant growth. J. Agric. Food Chem., 2003. 51, 6165-6172.

[11] Rimando, A. M.; Dayan, F. E.; Mikell, J. R.; Moraes, R. M. Phytotoxic lignans of Leucophyllum frutescens. Nat. Toxins, 1999, 7, 3943.

[12] Russel, G. B.; Singh, P.; Fenemore, P. G. Insect-control chemicals from plants. Toxic lignans from Libocedrus bidwillii. Aust. J. Biol. Sci., 1976, 29, 99-103.

[13] Oliva, A.; Moraes, R. M.; Watson, S. B.; Duke, S. O.; Dayan, F. E. Aryltetralin lignans inhibit plant growth by affecting the formation of mitotic microtubular organizing centers. Pestic. Biochem. Physiol., 2002, 72, 45-54.

[14] Deltoro, V. I.; Gimeno C.; Calatayud A.; Barreno E. Effects of $\mathrm{SO}_{2}$ fumigations on photosynthetic $\mathrm{CO}_{2}$ gas exchange, chlorophyll a fluorescence emission and antioxidant enzymes in the lichens Evernia prunastri and Ramalina farinacea. Physiologia Plant, 1999, 105, 648-654.

[15] Wellburn, A.R. The spectral determination of chlorophylls a and b, as well as total carotenoids, using various solvents with spectrophotometers of different resolution. J. Plant Physiol., 1994, 144, 307313.

[16] Bradford, M.M. A rapid and sensitive method for the quantification of microrganism quantities of protein utilizing the principle of protein dye binding. Anal. Biochem., 1976, 72, 248-254.

[17] Augusti, A.; Lauteri, M.; Spaccino, L.; Brugnoli, E. Short- and long- term responses of carbon isotope discrimination and photosynthetic energy dissipation to elevated $\mathrm{CO}_{2}$ concentration. In: Raschi, A., Vaccari, F.P., Miglietta, F. Eds., Ecosystem Response to $\mathrm{CO}_{2}$ : The MAPLE Results, Luxembourg, Office for Official Publications of the European Communities, 1999, pp. 117-132.

[18] Carillo, P.; Mastrolonardo, G.; Nacca, F.; Fuggi, A. Nitrate reductase in durum wheat seedlings as affected by nitrate nutrition and salinity. Funct. Plant Biol., 2005, 32, 209-219.

[19] Carillo, P.; Mastrolonardo, G.; Nacca, F.; Parisi, D.; Verlotta, A.; Fuggi, A. Nitrogen metabolism in durum wheat under salinity: accumulation of proline and glycine betaine. Funct. Plant Biol., 2008, 35, 412-426.

[20] Pietrini, F.; Iannelli, M. A.; Battistelli, A.; Moscatello, S.; Loreto, F.; Massacci, A. Effects on photosynthesis, carbohydrate accumulation and regrowth induced by temperature increase in maize genotypes with different sensitivity to low temperature. Aust. J. Plant Physiol., 1999, 26, 367-373.

[21] Cramer, M.D.; Gao, Z. F.; Lips, S. H. The influence of dissolved inorganic carbon in the rhizosphere on carbon and nitrogen metabolism in salinity-treated tomato plants. New Phytol., 1999, 142, 441-450.

[22] Gibon, Y.; Blaesing, O. E.; Hannemann, J.; Carillo, P.; Hoehne, M.; Hendriks, J. H. M.; Palacios-Rojas, N.; Cross, J.; Selbig, J.; Stitt, M. A robot-based platform to measure multiple enzyme activities in arabidopsis using a set of cycling assays: comparison of changes of enzyme activities and transcript levels during diurnal cycles and prolonged darkness. Plant Cell, 2004, 16, 3304-3325.

[23] Esposito, S.; Carillo, P.; Carfagna, S.; Martino Rigano, V.; Vona, V.; Rigano, C. Glucose-6P dehydrogenase activities in barley roots upon $\mathrm{NH}_{4}{ }^{+}$supply. In Nitrogen in a sustainable ecosystem: from the cell to the plant. Martins-Loução M.A. Lips, S.H. Backhuys Eds. Leiden, The Netherlands, 2000, pp. 129-132.

[24] Osmond, B.; Ramus, J.; Levavasseur, G.; Franklin, L. A.; Henley, W.S. Fluorescence quenching during photosynthesis and photoinhibition of Ulva rotundata. Planta, 1993, 190, 97-106.

[25] Weir, T. L.; Park, S. W.; Vivanco, J. M. Biochemical and physiological mechanisms mediated by allelochemicals. Curr. Opin. Plant Biol., 2004, 7, 472-479.

[26] Jang, J. C.; Sheen J. Sugar sensing in higher plants. Trends Plant Sci., 1997, 2, 208-214.

[27] Espineda, C. E.; Linford, A. S.; Devine, D.; Brusslan, J. A. The AtCAO gene, encoding chlorophyll a oxygenase, is required for chlorophyll b synthesis in Arabidopsis thaliana. Proc. Natl. Acad Sci. USA, 1999, 96(18), 10507-10511.

[28] Baroli, I.; Gutman, B. L.; Ledford, H. K.; Shin, J. W.; Chin, B. L.; Havaux, M.; Niyogi, K.K. Photo-oxidative Stress in a Xanthophylldeficient Mutant of Chlamydomonas. J. Biol. Chem., 2004, 279, 6337-6344.

[29] Donn, G.; Köcher, H. Inhibitors of glutamine synthetase. Böger, P., Wakabayashi, K., Hirai, K., Eds. In: Herbicide Classes in Development: Mode of Action, Targets, Genetic Engineering, Chemistry; Berlin, Heidelberg, New York, 2002, pp. 87-101.

[30] Wakabayashi, K.; Böger, P. Phytotoxic sites of action for molecular design of modern herbicides (Part 2): Amino acid, lipid and cell wall biosynthesis, and other targets for future herbicides. Weed Biol. Manag., 2004, 4, 59-70. 